\title{
Four-Factor Prothrombin Complex Concentrate to Reduce Post-Traumatic Hemorrhage in Patients with Major Trauma, the PROCOAG Trial: Study Protocol for a Randomized Multicenter Double-Blind Superiority Study
}

Pierre Bouzat ( $\square$ PBouzat@chu-grenoble.fr )

Université Grenoble Alpes: Universite Grenoble Alpes

Jean-Luc Bosson

Grenoble Alpes University

Jean-Stéphane David

Lyon University Hospital

Bruno Riou

Paris APHP

Jacques Duranteau

Paris APHP

Jean-François Payen

Grenoble Alpes University

\section{Research Article}

Keywords: Acute traumatic coagulopathy, prothrombin complex concentrate, severe trauma, massive transfusion

Posted Date: June 1st, 2021

DOI: https://doi.org/10.21203/rs.3.rs-389880/v1

License: (c) (i) This work is licensed under a Creative Commons Attribution 4.0 International License.

Read Full License 


\section{Abstract}

Background: Optimal management of severe trauma patients with active hemorrhage relies on adequate initial resuscitation. Early administration of coagulation factors improves post-traumatic coagulation disorders and four-factor prothrombin complex concentrate (PCC) might be useful in this context. Our main hypothesis is that four-factor PCC in addition to a massive transfusion protocol decrease blood product consumption at day one in severe trauma patients with major bleeding.

Methods This is a prospective, randomized, multicenter, double-blind, parallel, controlled superiority trial. Eligible patients are trauma patients with major bleeding admitted to a French level-I trauma centre. Patients randomized in the treatment arm receive $1 \mathrm{~mL} / \mathrm{kg}$ four-factor PCC within one-hour postadmission while patients randomized in the controlled group receive $1 \mathrm{~mL} / \mathrm{kg}$ of saline solution $0.9 \%$ as a placebo. The primary endpoint is the amount of blood products transfused in the first 24 hours postadmission (including red blood cells, frozen fresh plasma and platelets). The secondary endpoints are the amount of each blood product transfused in the first 24 hours, time to achieve prothrombin time ratio < 1.5 , time to hemostasis, number of thrombo-embolic events at 28 days, mortality at 24 hours and 28 days, number of intensive care unit (ICU)-free days, number of ventilator-free days during ICU stay, number of hospital-free days within the first 28 days, hospitalization status at day 28 , Glasgow outcome scale extended for patients with brain lesions on initial cerebral imaging, and cost of each strategy at day 8 and 28. Inclusions have started in December 2017 and are expected to be complete by June 2021.

Discussion: If PCC reduces total blood consumption at day one after severe trauma, this therapy, in adjunction to a classic massive transfusion protocol, may be used empirically on admission in patients at risk of massive transfusion to enhance coagulation. Moreover, this treatment may decrease blood product related complications and may improve clinical outcome after post-traumatic hemorrhage.

Trial registration: This study has been prospectively registered on clinical trial on July 14, 2017 NCT03218722

\section{Administrative Information}

Note: the numbers in curly brackets in this protocol refer to SPIRIT checklist item numbers. The order of the items has been modified to group similar items (see http://www.equator-network.org/reportingguidelines/spirit-2013-statement-defining-standard-protocol-items-for-clinical-trials/). 


\section{Title $\{1\}$}

\section{The PROCOAG trial, four-factor prothrombin complex concentrate in severe trauma patients with major bleeding: a randomized multicentre double-blind superiority study.}

Trial registration Clinical trial, NCT03218722

$\{2 \mathrm{a}$ and $2 \mathrm{~b}\}$.

Protocol version Date: February 6th 2019, Version: 6.0

$\{3\}$

Funding $\{4\}$

PROCOAG is an investigator-initiated trial, supported by a non-profit grant from the French Ministry of Health (PHRCI 2015, $\left.{ }^{\circ} 15-059\right)$. LFB (Les Ullis, France) distributes Kanokad ${ }^{\circledR}$ for free to participating centers.

Author details \{5a\} Pr. Pierre Bouzat and Pr. Jean-François Payen : Pôle Anesthésie-Réanimation, Centre Hospitalo-Universitaire Grenoble-Alpes, Grenoble, France

Pr. Jean-Luc Bosson : Univ. Grenoble Alpes, CNRS, Public Health department CHU Grenoble Alpes, TIMC-IMAG, 38000 Grenoble, France

Pr. Jean-Stéphane David : Service d’Anesthésie Réanimation, Centre HospitaloUniversitaire Lyon-Sud, Faculté de Médecine Lyon-Est, Université Claude Bernard Lyon 1, Lyon, France

Pr. Bruno Riou : Sorbonne Université, UMRS INSERM 116, IHU ICAN, et Service des urgences, Hôpital Pitié-Salpêtrière, Assistance Publique-Hôpitaux de Paris, Paris, France

Pr. Jacques Duranteau : Département d'Anesthésie-Réanimation, Hôpitaux Universitaires Paris Sud, Université Paris XI, Faculté de Médecine Paris-Sud, Le Kremlin-Bicêtre, France.

Name and contact information for the trial sponsor $\{5 b\}$
French Ministry of Health, GIRCI AURA, 3 Quai des Célestins, 69002 Lyon, France. $\mathrm{PHRCl} 2015, \mathrm{n}^{\circ} 15-059$

Role of sponsor $\{5 c\}$

Funders have no role in trial design, conduction, data collection, nor analysis. Kanokad® safety aggregated data are shared with LFB.

\section{Introduction}

\section{Background and rationale $\{6 a\}$}

Severe trauma is the leading cause of mortality in young adults in developed countries and is the third cause of overall death in the United States (1). Death occurs within the first 48 hours among $87 \%$ of trauma patients with fatal outcome and the first cause of death at 24-hour is massive bleeding $(2,3)$. Hemorrhagic shock is preventable with adequate strategies including early control of the active source of bleeding and an optimization of initial resuscitation called "damage control resuscitation" (4). The concept of damage control resuscitation is based on early administration of blood products, immediate treatment of post-traumatic coagulopathy and a reduction of crystalloid infusion. This strategy aims at treating hypovolemia and coagulation disorders while avoiding dilution coagulopathy (4). International 
guidelines for blood product administration recommend the transfusion of packed red blood cell (RBC) and fresh frozen plasma (FFP) as soon as possible with RBC/FFP ratio between 1:1 and 2:1 $(5,6)$.

Early administration of coagulation factors with FFP has been highlighted in the prehospital setting suggesting potential beneficial effect on mortality (7). However, FFP administration may be delayed due to logistical reasons such as transport and immediate availability. The administration of four-factor prothrombin complex concentrate (PCC) has emerged as an alternative to early administrate coagulation factors and is used to treat post-traumatic coagulopathy in diverse European countries $(8,9)$. A strategy combining fibrinogen and four factor-PCC has decreased blood product consumption (8). The reduction of blood product consumption is meaningful since blood transfusion is associated with post-traumatic infectious complications (10) and is a risk factor for multiple organ failure after severe trauma (11). Recently, an open-label randomized controlled trial comparing a classic massive transfusion protocol to a tailored administration of procoagulant concentrates, including PCC and fibrinogen, also showed a decrease in RBC transfusion resulting in less patients requiring massive transfusion (12). However, there is no randomized controlled double-blind study assessing the impact of PCC in addition to a classic massive transfusion protocol including fibrinogen administration and blood product transfusion with RBC and FFP (13). The PROCOAG study therefore aims to test whether early administration of PCC allows a reduction of total blood transfusion in trauma patients with major hemorrhage.

\section{Objectives $\{7\}$}

The primary objective of the PROCOAG study is to test whether the early PCC administration with standard blood products compared with standard care alone allows better control of post-traumatic hemorrhage, mirrored by the total blood product consumption at day one in trauma patients with major bleeding.

Secondary objectives are

- To conduct a separate analysis of the primary outcome:

- Assessment of RBC consumption

- Evaluation of FFP consumption

- Evaluation of Platelet consumption

- To compare between the two groups:

- Time to normalize hemostasis

- Time from admission to hemorrhage control defined during a surgical and/or radiological procedure.

- Thromboembolic events (pulmonary embolism, deep venous thrombosis)

- Morbi-mortality

$\circ$ Quality of life 
- To evaluate the economic benefit of the new strategy from the hospital's point of view.

\section{Trial design $\{8\}$}

The PROCOAG study is a therapeutic, prospective, randomized, multicenter, double-blind, parallel, superiority controlled clinical trial comparing the efficacy of four factor-PCC and a placebo (1:1 ratio) in controlling hemorrhage in patient at risk of massive transfusion.

\section{Methods: Participants, Interventions And Outcomes}

\section{Study setting $\{9\}$}

Twelve French academic hospitals participate in the study. All centers are Level-I trauma centers receiving more than 200 trauma patients with an injury severity score (ISS) $>15$ each year. List of participating centers can be found on https://ClinicalTrials.gov

\section{Eligibility criteria $\{10\}$}

\section{- Inclusion criteria}

Consecutive adult patients at risk of massive transfusion after severe trauma are screened for enrolment. Patients older than 18 years, admitted directly from the injury scene to a level-I trauma center are eligible provided that they have two risk factors : 1) a transfusion of at least one unit (U) of RBC prior to hospital admission or within the first hour after their admission (a delay of 90 min is tolerated if transfusion is decided within one hour post admission) and 2) an assessment of blood consumption (ABC) score $\geq 2$ (14) or clinical prediction of massive transfusion defined by a transfusion of at least 10 RBC during the first 24 hours or 3 RBC during the first hour.

\section{- Exclusion criteria}

Patients are not eligible if they show an exclusion criterion : 1) Traumatic cardiac arrest before randomization, 2) secondary admission from another health care facility/hospital (technical short stops are accepted), 3) treatment with anticoagulants, 4) known pregnancy, 5) known hypersensitivity to fourfactor PCC or its excipients, 6) patient with devastating injuries expected to die within the first hour postadmission, 7) patient under therapeutic limitation before randomization, 8) patient under guardianship, 9) knowledge of a contraindication to the use of $\mathrm{NaCl} 0.9 \%$ at the dose of $1 \mathrm{~mL} / \mathrm{kg}$ (e.g. hyperchloremia or hypernatremia), 10) treatment with an experimental medicine within the last 30 days, 11) family or patient refusal and 12) patient without health insurance.

\section{Who will take informed consent? $\{26 \mathrm{a}\}$}

Because of selection criteria, some enrolled patients are unable to consent (e.g. unconsciousness, agitation, post-traumatic stress). Informed consent can be therefore obtained from patient's next-of-kin if present or consent is waived if no one is present at the time of enrolment, as authorized by French law in 
emergency situation and approved by the ethics committee. This procedure was already used and authorized in a previous French study on trauma (15). As soon as the patient's next of kin is available and capable of informed consent in complete and faithful terms, consent is obtained. Informed consent must also be obtained from the patient whenever he/she becomes able to consent and up to 28 days after trauma. Patients and their next of kin have the right to refuse to participate in the study or revoke their consent at any time. All information appears in an information notice and a consent form given to the subject. All these documents have been approved by the ethics committee. Written informed consent is obtained by the investigator. Two original copies are co-signed by both the investigator and the subject who consent.

\section{Additional consent provisions for collection and use of participant data and biological specimens \{26b\}}

This is not applicable

\section{Interventions}

\section{Explanation for the choice of comparators $\{6 \mathrm{~b}\}$}

The comparator will be normal saline $(\mathrm{NaCl} 0.9 \%$, dose: $1 \mathrm{ml} / \mathrm{Kg})$. At this dose, $\mathrm{NaCl}$ is neutral regarding its interaction with the coagulation factors, and therefore is considered a placebo.

\section{Intervention description \{11a\}}

Within one hour after admission, patients are randomized in two arms. In placebo arm, patients are managed following standard care in addition to intravenous $1 \mathrm{ml} / \mathrm{kg}$ saline solution. In treatment arm, patients are managed following standard care in addition to intravenous $1 \mathrm{ml} / \mathrm{kg}$ four-factor PCC.

All centers follow European recommendations for managing hemorrhagic patients. Details of standard management are found below (11d).

\section{Criteria for discontinuing or modifying allocated interventions $\{11 \mathrm{~b}\}$}

Study treatment is managed by the investigating team, starting within one-hour post admission and is administered in intravenous perfusion at $2 \mathrm{~mL}$ per minute. There is no reason for discontinuing or modifying treatment unless adverse reaction to the study treatment occurs. In this unlikely event, perfusion should be stopped, appropriate care provided immediately and event should be reported to the sponsor. Dose change is not permitted by the protocol.

\section{Strategies to improve adherence to interventions $\{11 \mathrm{c}\}$}

Because the investigating team administrates the treatment, no strategy is needed to improve patient adherence to intervention.

Relevant concomitant care permitted or prohibited during the trial $\{11 \mathrm{~d}\}$ 
From admission, all patients are managed according to European recommendations. Briefly, the source of bleeding should be identified as soon as possible and treated with emergency surgery and/or embolization following standard practice (5). Regarding resuscitation, patients are treated in both groups with restricted volume replacement strategy, early transfusion of blood products with a RBC:FFP ratio between 1:1 and 2:1. Tranexamic acid (TXA) is intravenously administered within $3 \mathrm{~h}$ after injury at a loading dose of $1 \mathrm{~g}$ infused over $10 \mathrm{~min}$ followed by an intravenous infusion of $1 \mathrm{~g}$ over $8 \mathrm{~h} \mathrm{(16).}$ Fibrinogen concentrate is administered in case of fibrinogen concentration $<1.5 \mathrm{~g} / \mathrm{L}$ (17) or viscoelastic evidence of a functional fibrinogen deficiency (18). Platelets are administered to maintain a platelet count above $50 \times 10^{9} / \mathrm{L}$. Vasopressors can be infused in addition to fluids to maintain target systolic arterial blood pressure ( $>90 \mathrm{mmHg}$ or $>110 \mathrm{mmHg}$ for head injured patients). Blood samples are regularly drawn from admission to test hemostasis (prothrombin time ratio, Quick time, fibrinogen concentration and viscoelastic tests where available), blood electrolytes, hepatic and renal function and blood gases.

\section{Provisions for post-trial care $\{30\}$}

Post-trial care is not planned. Patients who suffer harm from trial participation will be cared for in the intensive care unit. Should prejudice linked to study participation occur, financial compensation will be provided by the insurance (Société Hospitalière d'Assurances Mutuelles-SHAM, 18 rue Edouard Rochet, 69,372 Lyon Cedex 08, France) contracted by the promotor.

\section{Outcomes $\{12\}$}

The primary outcome is the amount of blood products transfused in the first $24 \mathrm{~h}$ measured in number of units administered (and volume), including RBC, FFP and platelets. The choice of this primary outcome is based on: 1) recent randomized control trial regarding vasopressin use after severe trauma used the same endpoint (19), 2) even if transfusion is the main therapy to treat acute bleeding, blood products are also associated with adverse events and poor clinical outcome $(11,20)$ and 3$)$ mortality after severe trauma is a complex process depending on hemorrhage but also on traumatic brain injury and preexisting medical conditions, specifically in older patients (21). We thus believe that $24 \mathrm{~h}$ total blood consumption is an accurate proxy of the severity of the bleeding until the hemorrhage stops.

Secondary outcome measures are 1) number of units (and volume) of RBC, FFP and platelets taken separately and transfused in the first 24 hours, 2) time to achieve prothrombin time ratio $<1.5,3$ ) time to hemostasis, defined as the time necessary to control bleeding in the surgical field (operative field is dry without further foreseeable homeostatic procedures) or to control contrast blush after embolization during interventional radiology (no more dye flushing) 4) number of thrombo-embolic events at 28 days, 5) mortality at 24 hours and 28 days, 6 ) intensive care unit (ICU)-free days (number of in-hospital days outside ICU) within the first 28 days, 7) ventilator-free days during ICU stay within the first 28 days, 8) hospital-free days within the first 28 days, 9) hospitalization status at day 28,10 ) GOSE for patients with brain lesions on initial scan, and 11) cost of each strategy at day 8 and 28. Cost analyses will be 
performed on expensive care: blood products and procoagulant treatments administered, imaging exams, surgeries and length of stay in each healthcare unit.

\section{Participant timeline $\{13\}$}

All consecutive trauma patients admitted directly from the injury scene who require RBC transfusion with a risk of massive transfusion are screened on admission. Eligible patients are then rapidly enrolled by the study investigator. They should be randomized and treated accordingly within 60 minutes post admission. After patient treatment, follow-up includes hourly visits until $6 \mathrm{~h}$ post-admission, as well as visits at 12 and $24 \mathrm{~h}$ to measure prothrombin ratio, to assess bleeding control and to monitor adverse events. Thrombo-embolic events are under special scrutiny during the whole in-hospital stay because they are frequent expected adverse effects of PCC treatment. Each suspicious thrombo-embolic event should be confirmed with venous doppler echography or angiography and treated following standard practice. Thromboprophylaxis is left at the physician discretion. Patients are followed for 28 days with the last visit aiming at collecting the Glasgow outcome scale extended (GOSE) for patients with brain lesions on initial cerebral imaging, mortality and place of living (Figure 1).

\section{Sample size $\{14\}$}

Total blood consumption was estimated in the control group based on one-year consumption in Grenoble University hospital and Annecy General Hospital; we found that patients with our eligibility criteria received 12 units on average of RBC, FFP and Platelets. We consider a reduction of 3 units as clinically significant since 3 units represents 1 Liter of blood product. Thus, a $25 \%$ decrease in transfused blood products in the first $24 \mathrm{~h}$ is expected (from an average of 12 transfused units in the control group to 9 transfused units in the experimental group). Study needs to include 162 patients per group to demonstrate this $25 \%$ reduction with $80 \%$ power and a-risk of 0.05 , bilateral test (nQuery, Sample Size and Power Calculation, "Statsols", Statistical Solutions Ltd, Cork, Ireland, blood product units transfused do not follow normal distribution). To compensate estimated lost-to-follow up, the trial plans to include 350 patients.

\section{Recruitment $\{15\}$}

All centers are level-I trauma centers in France with subsequent recruitment of severe trauma patients. Before the start of the study, a feasibility questionnaire has circulated between all centers and they were asked for potential inclusions in the study. They all agree to include between one and two patients each month. Regular newsletters will be sent and all participating centers are part of a national network for trauma care (GITE group).

\section{Assignment of interventions: allocation}

\section{Sequence generation $\{16 a\}$}


Randomization will be performed by sealed envelopes available at the investigator site. Randomization will be by block of random size from 2 to 6 , stratified by center. The randomization list will be created and maintained by the study sponsor. Randomization envelopes to be opened will be created prior to the study by the coordinating pharmacy and made available during the site visit. The integrity and presence of the envelopes will be checked at each monitoring visit.

\section{Concealment mechanism \{16b\}}

Allocation sequence is implemented through sequentially numbered, opaque, sealed, envelopes. Envelopes have been created by the coordinating pharmacy in Grenoble and provided to each investigating site pharmacy which manages the stock. Site pharmacy provides 4 sequentially numbered envelopes to the investigating team and renew the stock after each randomization. Envelopes are sealed with inviolable tapes making any opening visible. Therefore, the investigating team cannot modify inclusion decision after allocation without being noticed by monitoring visits or the pharmacy.

\section{Implementation $\{16 c\}$}

One independent statistician generates the allocation sequence. Investigator (trained medical doctor in in the emergency room) enroll participants and an unblinded, trained nurse not involved in patient care assign participants to interventions by opening the appropriate randomization envelope.

\section{Assignment of interventions: Blinding}

\section{Who will be blinded $\{17 \mathrm{a}\}$}

After enrolment, the investigator contacts an unblinded, dedicated, trained nurse and transmits the patient's name, weight and study number. This nurse is not involved in patient care and does not belong to the trauma bay. The nurse opens the appropriated numbered envelope. Envelopes indicates the treatment arm, placebo (saline solution $0.9 \%$ ) or PCC, which is prepared accordingly at a dose of $1 \mathrm{ml} / \mathrm{kg}$ for both treatments, from a dedicated stock stored in a closed fridge box. Treatment is prepared into opaque syringes and then administered to the patient by the same dedicated nurse at $120 \mathrm{~mL} / \mathrm{h}$ with syringe pumps.

The investigating team and the patient are therefore blinded (investigator and any MD implied in patient care, nurses, site clinical research assistants). The dedicated nurse is unblinded.

Several strategies aim at securing masking: the dedicated nurse is not involved in patient care and this is carefully verified during monitoring visits, perfusion material (syringes, filling pipes and dressings) is opaque, and places for treatments storage, randomization and treatment preparation are dedicated areas, apart from the patient care area.

Site pharmacy and pharmacy site monitor are not blinded. Statistician and data monitor are blinded to treatment allocation. 


\section{Procedure for unblinding if needed $\{17 b\}$}

Unblinding will be performed only when knowledge of the treatment allocated to the patient is necessary for his/her medical management. The investigator, who needs to know the group in which his/her patient is randomized, will be able to contact the pharmacy of his/her center.

At the time of the call, the referring person will have to write on a standard document:

- The contact details of the physician, investigator or not, or the pharmacist who called (name, first name, qualification of the caller, address, telephone number).

- The name of the study.

- The date of the call, the description of the clinical event motivating the call: type of event, start date, treatment modalities.

- If the event is classified as a serious adverse event.

- The action following this call: unblinding, action to be taken to explore the effect and allow diagnosis, treatment of the effect.

The randomization list is stored in the clinical pharmacy of each investigative center.

This information will be sent as soon as possible to the study sponsor by e-mail.

The vigilance unit will be systematically unblinded in case of a suspected unexpected serious adverse reaction before the reporting to the competent authorities, and possibly annually when the annual safety reports are drawn up.

\section{Data collection and management}

\section{Plans for assessment and collection of outcomes $\{18 \mathrm{a}\}$}

An electronic case report form (eCRF, CSonline by clinsight) will be created for the study. The persons responsible for completing the eCRF will be clearly identified in the task delegation document.

Grenoble University Hospital is the sponsor of the study and is responsible for study oversight and quality controls. Data are collected by the investigating centers staff on the electronic case report form. Anonymized data are stored by and belongs to the sponsor. Investigating sites are monitored by a dedicated department, independent of the scientific team and investigators who designed the project. Centers are visited regularly to control patient data, ethics, patient safety, respect of masking protocol and investigational products handling and storage. Primary endpoint is monitored in all patients.

All visits will be the subject of a monitoring report in writing. A copy will be sent to the Principal Investigator.

Plans to promote participant retention and complete follow-up $\{18 \mathrm{~b}\}$ 
The primary outcome is collected at Day one (total blood consumption). This endpoint is easily checked through blood bank data. Follow-up lasts up to 28 Days; considering the high severity of the patients and their admission in ICU, no specific action is planned to promote participant retention.

\section{Data management $\{19\}$}

Data management will follow Grenoble-Alpes university hospital procedures (data validation, basic freezing, backup, data reviews before unblinding). A data entry dictionary will be made available to investigators and their teams and traceability of requests for corrections and corrections made on the eCRF.

\section{Confidentiality $\{27\}$}

In accordance with the law (Articles L.1121-3 and R.5121-13 of the Public Health Code), persons with direct access to data will take all necessary precautions to ensure the confidentiality of information relating to experimental drugs, research, and the persons involved, particularly with regard to their identity and the results obtained. These persons, in the same way as the investigators themselves, are subject to professional confidentiality.

During or at the end of the interventional research, the data collected on patients and transmitted to the sponsor by the investigators will be made anonymous. Under no circumstances should the names or addresses of the patients be made clear. Only the first letter of the subject's surname and first name will be recorded, accompanied by a coded number specific to the research, indicating the order of inclusion of the subjects.

The promoter will ensure that each person who participates in the research has given written consent for access to individual data concerning him or her that is strictly necessary for the quality control of the research.

\section{Plans for collection, laboratory evaluation and storage of biological specimens for genetic or molecular analysis in this trial/future use $\{33\}$}

This is not applicable

\section{Statistical methods}

\section{Statistical methods for primary and secondary outcomes $\{20 \mathrm{a}\}$}

The risk of alpha error is the usual threshold: $5 \%$. All our tests will be bilateral. The initial characteristics of the patients will be represented in a comparative table according to the two treatment groups. Quantitative and qualitative variables will be presented with the usual parameters, mean and standard deviation as well as numbers and percentages.

Analysis of the main outcome: 
The main outcome is the total blood consumption (RBC, FFP and Platelets). It will be analyzed after verification that the time spent in the study up to $\mathrm{H} 24$ is not significantly different between the two groups. If this duration is not different, the total number of units transfused over 24 hours between the two groups will be compared using a bilateral student intent to treat t-test (if the application conditions are not met, we will use a non-parametric Mann-Whitney U-test). If the duration is different, the hourly densities of blood products between the two groups will be compared using an intention-to-treat student t-test (= total number of bags transfused over 24 hours divided by the time spent in research during the first 24 hours), in order to control for an attrition bias created by patients who died before 24 hours.

The per-protocol analysis will be performed on patients who actually received treatment within one hour of admission to the hospital.

Secondary outcome analysis:

We will use the same strategy as for the primary endpoint in order to compare each quantity of RBC, FFP and Platelets, independently; i.e., a Student's t-test (if the application conditions are not met, we will use a non-parametric test).

Comparison by a Student t-test (if the application conditions are not met, we will use a nonparametric test) of the values of the Quick Time between the two groups.

The delays between trauma and bleeding cessation will be compared between the two randomization groups by a Student t-test (if the application conditions are not met, we will use a nonparametric test).

The number of thromboembolic events at D28 will be recoded as a qualitative variable. At least one event (pulmonary embolism or deep vein thrombosis) will be required for this variable. It will then be compared using the Chi-Two test between the two groups (when the conditions for application of the Chi-Two test are not met, the Fisher test will be used).

Calculation of the cost of each of the strategies:

An average cost of care will be calculated for each group. Micro-costing and gross-costing methods will be used to reconstitute the cost of the hospital stay. Thus, data on the consumption of care will be collected prospectively throughout the entire length of the stay in intensive care (the number and nature of blood products and drug treatments used, additional examinations performed, as well as the length of stay in each care unit). In this way, we can calculate an average cost of the resuscitation stay in each group. The cost of the overall stay will be reconstituted using the modified GHM method, it will be based on hospital cost accounting data (calculated within the framework of the ENC: national scale of costs).

Comparison of the cost of the two strategies: The difference in costs between the groups will be tested by a Student test or by a non-parametric test (Mann-Whitney tests) in case of non-Gaussian distribution. The choice of the test will be made with regard to the distribution of costs in each group (Shapiro-Wilk test, on raw data or, if necessary, on transformed data). If the distribution of costs is not Gaussian, the 
construction of the confidence interval of the average cost will be based on the non-parametric bootstrap method.

Deterministic and probabilistic sensitivity analyses will be performed to test the robustness of our estimates. Multivariate analyses will also be implemented to take into account censoring (death and follow-up time) in our cost analyses.

Mortalities at 24 hours and 28 days will be compared between the two groups using the Kaplan-Meier model.

Length of stay will be compared with a Student t-test (if the application conditions are not met, we will use a non-parametric test).

The GOSE at 28 days will be compared with a Student's t-test (if the application conditions are not met, we will use a non-parametric test). The locations of the patient's hospitalization at 28 days will be compared between the two groups using the Chi-Two test (when the application conditions of the ChiTwo test are not met, the Fisher test will be used).

\section{Interim analyses $\{21 \mathrm{~b}\}$}

No interim analysis is planned

\section{Methods for additional analyses (e.g. subgroup analyses) \{20b\}}

Since eligibility criteria may select patients without massive bleeding due to imperfect accuracy of the $A B C$ score or wrong clinical judgement, primary and secondary outcomes will also be tested in the population with a massive transfusion (3 RBC within the first post-traumatic hour or at least $10 \mathrm{RBC}$ at Day one).

\section{Methods in analysis to handle protocol non-adherence and any statistical methods to handle missing data $\{20 \mathrm{c}\}$}

Analysis will be made following an intention-to-treat principle and a per-protocol principle to handle protocol non-adherence. We expect few missing data on the primary outcome since total blood consumption is always recorded and followed by the blood bank in each center.

\section{Plans to give access to the full protocol, participant level-data and statistical code $\{31 \mathrm{c}\}$}

Information from the full protocol will be published in a peer-reviewed journal and the study is registered in ClinicalTrials.gov. The relevant data analyzed during the development of this study protocol are available upon request from the corresponding author.

\section{Oversight and monitoring}


Coordinating center: Grenoble University Hospital is the sponsor of the study and is responsible for study oversight and quality controls.

Steering committee: Pierre Bouzat, Jean-Stephane David, Jean-Luc Bosson, Bruno Riou, Jacques Duranteau, Jean-François Payen

Data management team: Jean-Luc Bosson, MD, PhD, CHU Grenoble Alpes, Grenoble, France

\section{Investigational products handling and storage:}

Shipping of investigational products is under the responsibility of the coordinating clinical trial pharmacy of Grenoble University Hospital. Specifically, they create and ship randomization envelopes, they order PCC (Kanokad@) to the manufacturer LFB (Les Ullis, France). The biopharmaceutical company LFB is responsible for producing and delivering Kanokad $\$$ to investing sites in respect with Good Manufacturing Practices. The coordinating pharmacy in Grenoble also orders and distributes saline solution and opaque devices required for the research to investigating sites. Each site is responsible for storage $\left(2-8{ }^{\circ} \mathrm{C}\right.$, away from light), handling and accounting of investigational products received. These elements are verified during monitoring visits.

\section{Composition of the data monitoring committee, its role and reporting structure $\{21 a\}$}

The sponsor appointed a data safety monitoring board (DSMB) that meets every year to analyze safety data (expected and unexpected adverse events). The DSMB is composed of members independent of the research and make recommendations about protocol termination, modifications or continuation without modifications.

\section{Adverse event reporting and harms $\{22\}$}

Adverse events are reported blinded to the sponsor by investigating sites and are analysed by the sponsor safety departments following European regulation on clinical trials on medicinal products.

\section{Frequency and plans for auditing trial conduct $\{23\}$}

An audit carried out at the request of the sponsor or an inspection by the health authorities may be conducted at any time by persons independent of those responsible for the research. Its purpose is to ensure the quality of the research, the validity of its results and compliance with the law and regulations. The auditors/inspectors must have direct access to the source and medical data and to any useful document related to the conduct of the clinical study. The confidentiality of the data and the anonymity of the subjects will then be respected.

The investigators agree to comply with the sponsor's and the competent authority's requirements for an audit or inspection of the research. 
The audit may apply to all stages of the research, from the development of the protocol to the publication of results and the classification of data used or generated in the research.

\section{Plans for communicating important protocol amendments to relevant parties (e.g. trial participants, ethical committees) $\{25\}$}

Any substantial modification, i.e. any modification likely to have a significant impact on the protection of persons, on the conditions of validity and on the results of the research, on the quality and safety of the products tested, on the interpretation of the scientific documents that support the conduct of the research or on the methods of conducting the research, is the subject of a written amendment that is submitted to the sponsor; the latter must obtain, prior to its implementation, a favorable opinion from the ethic committee and an authorization from the national agency for drug safety. All amendments are validated by the sponsor and by all the research stakeholders concerned by the amendment before submission to the ethic committee and the national agency for drug safety. All amendments to the protocol must be made known to all investigators participating in the research. The investigators undertake to respect the content of the protocol. Any amendment that modifies the management of the subjects or the benefits, risks and constraints of the research is the subject of a new information note and a new consent form, the collection of which follows the same procedure as above.

\section{Dissemination plans $\{31$ a $\}$}

Results will be reported through scientific conferences and peer-reviewed publications following CONSORT recommendations (22). All participants can be informed of the study results at the end of the trial on request.

\section{Discussion}

The PROCOAG trial proposes to manage post-traumatic coagulopathy using pro-thrombin concentrate complex (PCC) in addition to standard blood products and fibrinogen. As opposed to fresh frozen plasma, PCC is readily available at admission and we hypothesized that early correction of coagulation factor deficiency optimizes patient blood management and therefore reduce blood products transfused within the first 24 hours following a severe trauma.

Hemorrhage is a leading cause of death in patients with trauma. Although blood products remain the standard for treating hemorrhagic shock, they are a limited and perishable resource. Moreover, concerns are increasing that blood products are immunomodulatory and may negatively affect clinical outcomes (23-25). Resuscitation strategies that decrease the need for transfusions without increasing complications, therefore, would represent a clinically important innovation.

Eligibility criteria were chosen to early select patients with severe acute bleeding so that PCC can be early administrated in the trauma bay as soon as the patient arrived. We are aware that some patients with acute hemorrhage may not require massive transfusion but the study has to be pragmatic and the main 
hypothesis is that an empiric administration of PCC may enhance coagulation and then decrease total blood consumption. Moreover, even if some patients included don't require massive transfusion, planned sub-group analysis will help to overcome this effect.

The authors also acknowledge that total blood transfusion is only a surrogate marker of the severity of the bleeding. However, the AVERT Shock trial also successfully used this endpoint considering that the reduction of blood transfusion is one of the modern objectives of trauma resuscitation (19). The amount of transfusion has been shown to correlate with severe complications and poor clinical outcome $(10,20$, 26). Specifically, Moore et al noted that transfusion of packed red blood cells was the strongest predictor for the development of multi-organ failure (Odds Ratio $=8.6)(11)$. The strategy tested in the present trial hence carries the potential to reduce morbidity, mirrored by the length of stay in ICU and the duration of mechanical ventilation, by reducing the amount of used blood products. Another limitation is that, although following guidelines, centers may have variations in standard hemorrhage management. However, randomization stratification on centers should correct for a potential center effect.

As a pragmatic study, this trial aims at testing the effect of adding PCC to standard care in real life situations. With high level of proof and quality standard, a positive result might already affect management of severe trauma patients at risk of massive transfusion as this study investigates PCC efficacy, safety and cost in this indication.

\section{Trial status}

The trial has obtained all authorizations and recruitment started in December 2017. Enrollment is expected to be complete by June 2021. Protocol version reported here is version 6 as of February $6^{\text {th }}$, 2019.

\section{Abbreviations}

DSMB: data safety monitoring board; eCRF: electronic case report form; FFP: Fresh Frozen Plasma; GOSE: Glasgow Outcome Scale Extended; ICU: Intensive Care Unit; NaCl: normal saline; PCC: Prothrombin Complex Concentrate; RBC: Red Blood Cell

\section{Declarations}

\section{Acknowledgements}

We thank patients and their relatives, physicians, nurses and clinical research staff of the participating centres for their involvement in this study. We thank Dr. Audrey Lehmann, Anaïs Adolle, Pauline Manhes, Marion Richard, Aline Baron, and Jennifer Margier for their involvement in study design and/or study oversight. 
PROCOAG study group : Annecy-Gennevois Hospital, Annecy, France (Dr. Albrice Levrat), AP-HP Beaujon, Clichy, France (Dr. Paër-Selim Abback), AP-HP Bicêtre, Le Kremlin-Bicêtre, France (Pr. Jacques Duranteau), AP-HP Pitié Salpêtrière, Paris, France (Pr. Bruno Riou), Lille University Hospital, Lille, France (Dr. Delphine Garrigue), HCL - Hôpital Edouard Herriot, Lyon, France (Dr. Guillaume Marcotte), HCL - Lyon Sud, PierreBénite, France (Pr. Jean-Stéphane David), Montpellier University Hospital, Montpellier, France (Dr. Jonathan Charbit), Nantes University Hospital, Nantes, France (Pr. Karim Asehnoune), AP-HM - Marseille Nord, Marseille Nord (Pr. Marc Leone), Strasbourg University Hospital, Strasbourg, France (Pr. Julien Pottecher), Grenoble-Alpes University Hospital, Grenoble, France (Pr. Pierre Bouzat).

\section{Authors' contributions $\{31 \mathrm{~b}\}$}

PB and JFP designed the trial. JLB was in charge of the statistical plan. JSD, BR and JD extensively revised the protocol. All the authors approved the final version

\section{Funding $\{4\}$}

PROCOAG is an investigator-initiated trial, supported by a non-profit grant from the French Ministry of Health (PHRCI 2015, n¹5-059). LFB (Les Ullis, France) distributes Kanokad® for free to participating centers. These two funders have no role in trial design, conduction, data collection, nor analysis. Kanokad@ safety aggregated data are shared with LFB.

\section{Availability of data and materials $\{29\}$}

Only the principal investigators, the DSMB and the statisticians will have access to the final data set.

There is no plan to share data but they are made available for audits and inspections. Anonymized patient data or full set can be made available on reasonable request. Patients or their relative are informed that anonymized data can be shared with other groups or used for further studies and have the opportunity to refuse personal data sharing.

\section{Ethics approval and consent to participate $\{24\}$}

Protocol was approved by the Institutional Review Board Sud-Ouest et Outre Mer II (SOOM II), on 04/05/2017 and authorized by the French competent authority (Agence Nationale pour la Sécurité du Médicament, ANSM) on 21/04/2017 (documents provided as additional files). The study is carried out following Good Clinical Practices, the principles of the declaration of Helsinki and European legislation (27). Because of selection criteria, some enrolled patients are unable to consent (e.g. unconsciousness, agitation, post-traumatic stress). Informed consent can be therefore obtained from patient's next-of-kin if present or consent is waived if no one is present at the time of enrolment, as authorized by French law in emergency situation and approved by the ethics committee. This procedure was already used and authorized in a previous French study on trauma (15). As soon as the patient's next of kin is available and capable of informed consent in complete and faithful terms, consent is obtained. Informed consent must also be obtained from the patient whenever he/she becomes able to consent and up to 28 days after 
trauma. Patients and their next of kin have the right to refuse to participate in the study or revoke their consent at any time. All information appears in an information notice and a consent form given to the subject. All these documents have been approved by the ethics committee. Written informed consent is obtained by the investigator. Two original copies are co-signed by both the investigator and the subject who consent.

\section{Consent for publication $\{32\}$}

Although it is not anticipated, owing to the trial design, publication of any personal information about a patient will require her/his consent.

\section{Competing interests $\{28\}$}

PB, JSD, BR, JD and JFP received payments from LFB, Les Ullis, France for lectures and educational content.

\section{References}

1. Minei JP, Schmicker RH, Kerby JD, Stiell IG, Schreiber MA, Bulger E, et al. Severe traumatic injury: regional variation in incidence and outcome. Ann Surg. 2010;252(1):149-57.

2. Evans JA, van Wessem KJ, McDougall D, Lee KA, Lyons T, Balogh ZJ. Epidemiology of traumatic deaths: comprehensive population-based assessment. World J Surg. 2010;34(1):158-63.

3. Tisherman SA, Schmicker RH, Brasel KJ, Bulger EM, Kerby JD, Minei JP, et al. Detailed description of all deaths in both the shock and traumatic brain injury hypertonic saline trials of the Resuscitation Outcomes Consortium. Ann Surg. 2015;261(3):586-90.

4. Holcomb JB, Jenkins D, Rhee P, Johannigman J, Mahoney P, Mehta S, et al. Damage control resuscitation: directly addressing the early coagulopathy of trauma. J Trauma. 2007;62(2):307-10.

5. Rossaint R, Bouillon B, Cerny V, Coats TJ, Duranteau J, Fernandez-Mondejar E, et al. The European guideline on management of major bleeding and coagulopathy following trauma: fourth edition. Crit Care. 2016;20:100.

6. Holcomb JB, Tilley BC, Baraniuk S, Fox EE, Wade CE, Podbielski JM, et al. Transfusion of plasma, platelets, and red blood cells in a 1:1:1 vs a 1:1:2 ratio and mortality in patients with severe trauma: the PROPPR randomized clinical trial. JAMA. 2015;313(5):471-82.

7. Sperry JL, Guyette FX, Brown JB, Yazer MH, Triulzi DJ, Early-Young BJ, et al. Prehospital Plasma during Air Medical Transport in Trauma Patients at Risk for Hemorrhagic Shock. N Engl J Med. 2018;379(4):315-26.

8. Schochl H, Nienaber U, Maegele M, Hochleitner G, Primavesi F, Steitz B, et al. Transfusion in trauma: thromboelastometry-guided coagulation factor concentrate-based therapy versus standard fresh frozen plasma-based therapy. Crit Care. 2011;15(2):R83. 
9. Schochl H, Nienaber U, Hofer G, Voelckel W, Jambor C, Scharbert G, et al. Goal-directed coagulation management of major trauma patients using thromboelastometry (ROTEM)-guided administration of fibrinogen concentrate and prothrombin complex concentrate. Crit Care. 2010;14(2):R55.

10. Claridge JA, Sawyer RG, Schulman AM, McLemore EC, Young JS. Blood transfusions correlate with infections in trauma patients in a dose-dependent manner. Am Surg. 2002;68(7):566-72.

11. Moore FA, Moore EE, Sauaia A. Blood transfusion. An independent risk factor for postinjury multiple organ failure. Arch Surg. 1997;132(6):620-4. discussion 4-5.

12. Innerhofer $P$, Fries $D$, Mittermayr M, Innerhofer N, von Langen $D$, Hell $T$, et al. Reversal of traumainduced coagulopathy using first-line coagulation factor concentrates or fresh frozen plasma (RETIC): a single-centre, parallel-group, open-label, randomised trial. Lancet Haematol. 2017;4(6):e258-e71.

13. Joseph B, Aziz H, Pandit V, Hays D, Kulvatunyou N, Yousuf Z, et al. Prothrombin complex concentrate versus fresh-frozen plasma for reversal of coagulopathy of trauma: is there a difference? World $\mathrm{J}$ Surg. 2014;38(8):1875-81.

14. Nunez TC, Voskresensky IV, Dossett LA, Shinall R, Dutton WD, Cotton BA. Early prediction of massive transfusion in trauma: simple as ABC (assessment of blood consumption)? J Trauma. 2009;66(2):346-52.

15. Payen JF, Berthet M, Genty C, Declety P, Garrigue-Huet D, Morel N, et al. Reduced mortality by meeting guideline criteria before using recombinant activated factor VII in severe trauma patients with massive bleeding. Br J Anaesth. 2016;117(4):470-6.

16. collaborators C-t, Shakur, Roberts H, Bautista I, Caballero R, Coats J. T, et al. Effects of tranexamic acid on death, vascular occlusive events, and blood transfusion in trauma patients with significant haemorrhage (CRASH-2): a randomised, placebo-controlled trial. Lancet. 2010;376(9734):23-32.

17. Bouzat $P$, Ageron FX, Charbit J, Bobbia X, Deras P, Nugues JBD, et al. Modelling the association between fibrinogen concentration on admission and mortality in patients with massive transfusion after severe trauma: an analysis of a large regional database. Scand J Trauma Resusc Emerg Med. 2018;26(1):55.

18. Bouzat P, Guerin R, Boussat B, Nicolas J, Lambert A, Greze J, et al. Diagnostic performance of thromboelastometry in trauma-induced coagulopathy: a comparison between two level I trauma centres using two different devices. Eur J Trauma Emerg Surg. 2019.

19. Sims CA, Holena D, Kim P, Pascual J, Smith B, Martin N, et al. Effect of Low-Dose Supplementation of Arginine Vasopressin on Need for Blood Product Transfusions in Patients With Trauma and Hemorrhagic Shock: A Randomized Clinical Trial. JAMA Surg. 2019;154(11):994-1003.

20. Hill GE, Frawley WH, Griffith KE, Forestner JE, Minei JP. Allogeneic blood transfusion increases the risk of postoperative bacterial infection: a meta-analysis. J Trauma. 2003;54(5):908-14.

21. Sammy I, Lecky F, Sutton A, Leaviss J, O'Cathain A. Factors affecting mortality in older trauma patients-A systematic review and meta-analysis. Injury. 2016;47(6):1170-83. 
22. Moher D, Hopewell S, Schulz KF, Montori V, Gotzsche PC, Devereaux PJ, et al. CONSORT 2010 explanation and elaboration: updated guidelines for reporting parallel group randomised trials. Int $\mathrm{J}$ Surg. 2012;10(1):28-55.

23. Al-Refaie WB, Parsons HM, Markin A, Abrams J, Habermann EB. Blood transfusion and cancer surgery outcomes: a continued reason for concern. Surgery. 2012;152(3):344-54.

24. Glance LG, Dick AW, Mukamel DB, Fleming FJ, Zollo RA, Wissler R, et al. Association between intraoperative blood transfusion and mortality and morbidity in patients undergoing noncardiac surgery. Anesthesiology. 2011;114(2):283-92.

25. Turan A, Yang D, Bonilla A, Shiba A, Sessler DI, Saager L, et al. Morbidity and mortality after massive transfusion in patients undergoing non-cardiac surgery. Can J Anaesth. 2013;60(8):761-70.

26. Offner PJ, Moore EE, Biffl WL, Johnson JL, Silliman CC. Increased rate of infection associated with transfusion of old blood after severe injury. Arch Surg. 2002;137(6):711-6. discussion 6-7.

27. Toulouse E, Masseguin C, Lafont B, McGurk G, Harbonn A. J AR, et al. French legal approach to clinical research. Anaesth Crit Care Pain Med. 2018;37(6):607-14.

\section{Figures}




\section{Patient at risk of massive transfusion after severe trauma}

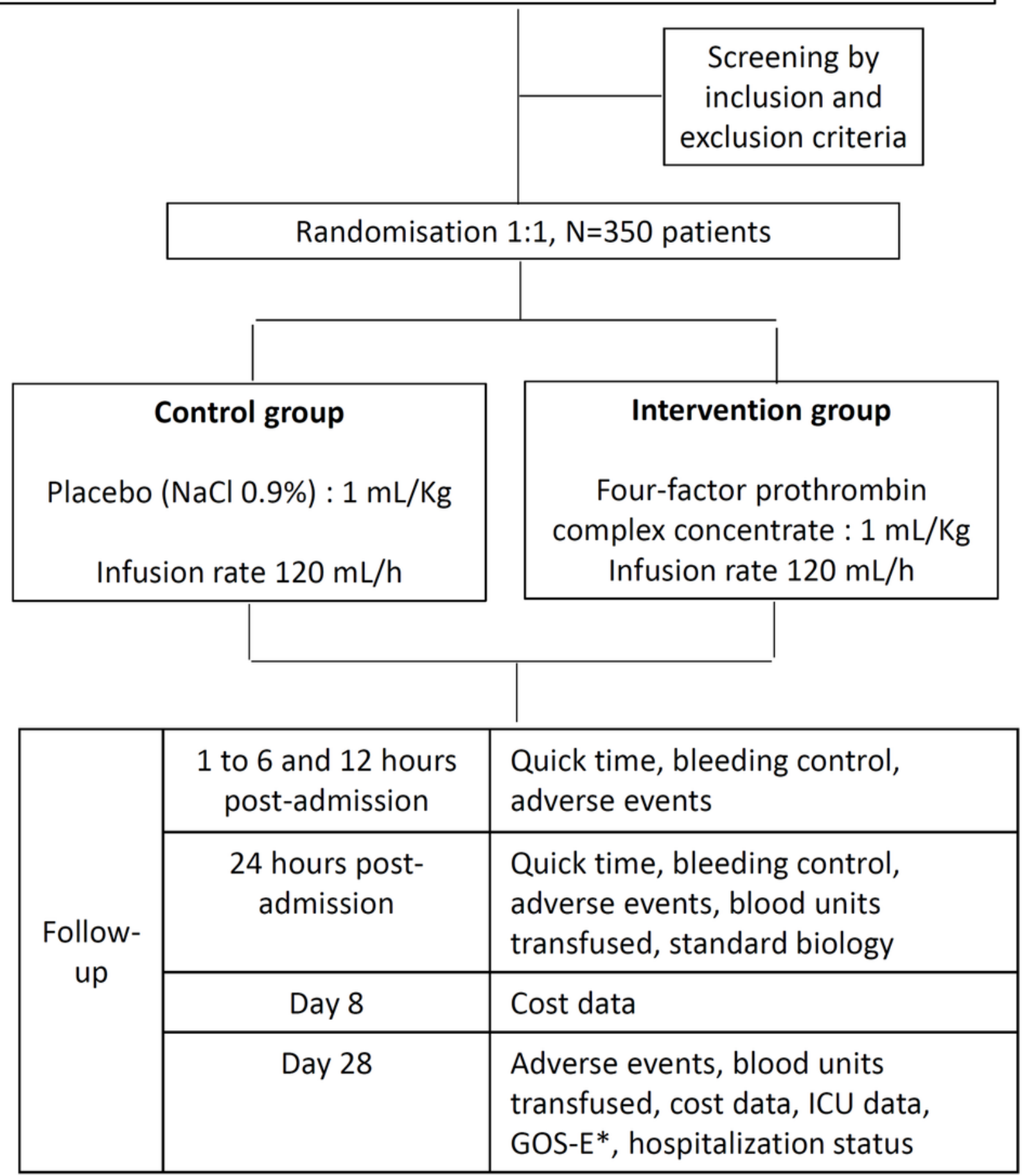

Figure 1

Participant timeline 